\title{
HARDY TYPE INEQUALITIES FOR AHARONOV-BOHM MAGNETIC POTENTIALS WITH MULTIPLE SINGULARITIES
}

\author{
A. A. Balinsky
}

Abstract. The aim of this article is to extend the Laptev-Weidl inequalities to the case of Aharonov-Bohm magnetic potentials with multiple singularities.

\section{Introduction}

It is known that the classical Hardy inequality

$$
\int_{\mathbb{R}^{n}} \frac{|u(\mathbf{x})|^{2}}{|\mathbf{x}|^{2}} d \mathbf{x} \leq \text { const. } \int_{\mathbb{R}^{n}}|\nabla u(\mathbf{x})|^{2} d \mathbf{x}, \quad u \in C_{0}^{\infty}\left(\mathbb{R}^{n} \backslash\{0\}\right)
$$

does not hold for $n=2$. In [3] Laptev and Weidl discovered that introducing a magnetic field can improve this situation. In particular, if the gradient $\nabla$ is replaced by the "magnetic" gradient $\nabla+i A$, where $A$ is a smooth magnetic vector potential in $\mathbb{R}^{2} \backslash\{0\}$ with

$$
B:=\operatorname{curl} A=0,
$$

then, for all $u \in C_{0}^{\infty}\left(\mathbb{R}^{2} \backslash\{0\}\right)$,

$$
\int_{\mathbb{R}^{2}} \frac{|u(\mathbf{x})|^{2}}{|\mathbf{x}|^{2}} d \mathbf{x} \leq C \int_{\mathbb{R}^{2}}|(\nabla+i A) u(\mathbf{x})|^{2} d \mathbf{x}
$$

with the sharp constant $C=\left(\min _{k \in \mathbb{Z}}|k-\Psi|\right)^{-2}$. Here $\Psi$ is the circulation of $A$ round the origin

$$
\Psi=\frac{1}{2 \pi} \oint_{\mathbb{S}^{1}} A d \mathbf{x}
$$

In this paper we are interested in similar inequalities for magnetic Dirichlet forms with Aharonov-Bohm vector potentials that have multiple singularities. Let $P_{1}=\left(x_{1}, y_{1}\right), \ldots, P_{n}=\left(x_{n}, y_{n}\right)$ be $n$ different points in $\mathbb{R}^{2}$. We can identify $\mathbb{R}^{2}$ with $\mathbb{C}$ by the correspondence $(x, y) \mapsto z=x+i y$ and the points $P_{1}, \ldots, P_{n}$ then correspond to the complex numbers $z_{1}=x_{1}+i y_{1}, \ldots, z_{n}=x_{n}+i y_{n}$. Consider a smooth vector potential $A=\left(A_{1}(x, y), A_{2}(x, y)\right)$ in the punctured

Received October 8, 2002.

Keywords and phrases. magnetic field, Hardy's inequality, Aharonov-Bohm potential.

2000 Mathematics Subject CLassification. Primary: 35R45, 35J10; Secondary: 81Q10, $35 \mathrm{Q} 40$. 
plane $M=\mathbb{R}^{2} \backslash\left\{P_{1}, \ldots, P_{n}\right\}$ with zero magnetic field (1.2) and denote by $\omega_{A}$ the differential 1-form $A_{1}(x, y) d x+A_{2}(x, y) d y$. Then (1.2) says that $\omega_{A}$ is a closed differential form in $M$, i.e. $d \omega_{A}=0$. Such a vector potential $A$ is known as a magnetic vector potential of Aharonov-Bohm type. The condition (1.2) implies that in any simply connected, open subset of $M$, there exists a gauge function $f$ such that $A=\nabla f$.

For each point $P_{k}(k=1, \ldots, n)$ let us define a circulation of $A$ round $P_{k}$ as

$$
\Phi_{k}=\frac{1}{2 \pi} \oint_{\gamma_{k}} A_{1}(x, y) d x+A_{2}(x, y) d y,
$$

where $\gamma_{k}$ is a small circle in $M$ which winds once around $P_{k}$ in an anticlockwise direction. Condition (1.2) implies that (1.5) is invariant under continuous deformations of $\gamma_{k}$. Furthermore, if the circulations $\Phi=\left(\Phi_{1}, \ldots, \Phi_{n}\right)$ of two distinct Aharonov-Bohm type vector potentials $A$ and $A^{\prime}$ in $M$ are equal modulo $\mathbb{Z}^{n}$ then $A$ and $A^{\prime}$ are equivalent under some gauge transformation $\phi: M \rightarrow U(1)$, i.e. $A^{\prime}=A+\frac{1}{i} \phi^{-1} \nabla \phi$. Here $U(1)=\{z \in \mathbb{C}|| z \mid=1\}$.

For any magnetic vector potential $A$ satisfying (1.2) in $M$ there exists a gauge function $f$ such that

$$
A(x, y)-\sum_{j=1}^{n} \frac{\Phi_{j}}{r_{j}^{2}} \cdot\left(-y+y_{j}, x-x_{j}\right)=(\nabla f)(x, y),
$$

where $r_{j}^{2}=\left(x-x_{j}\right)^{2}+\left(y-y_{j}\right)^{2}$ and $\Phi_{j}$ is the circulation of $A$ round $P_{j}$.

Given the vector potential $A$, we define the corresponding magnetic Dirichlet form on $C_{0}^{\infty}(M)$ by

$$
\mathcal{Q}_{A}[u]=\int_{M}|(\nabla+i A) u|^{2} d x d y, \quad u \in C_{0}^{\infty}(M) .
$$

Our main goal in this paper is to find an estimation from below for (1.6) by a Hardy-type expression

$$
\mathcal{Q}_{A}[u] \geq \int_{M} H(x, y)|u(x, y)|^{2} d x d y, \quad u \in C_{0}^{\infty}(M)
$$

with a suitable nonnegative function $H(x, y)$ on $M$.

For any real number $\Psi$ denote by $p(\Psi)$ the distance from $\Psi$ to the set of integers $\mathbb{Z}$, i.e.

$$
p(\Psi)=\min _{k \in \mathbb{Z}}|k-\Psi| .
$$

There are many functions $H(x, y)$ that give the inequality (1.7). We are interested in those $H(x, y)$ that satisfy the following conditions.

1. $H(x, y)$ depends on $A$ only throughout the circulations $\Phi_{1}, \Phi_{2}, \ldots, \Phi_{n}$ and the coordinates of $P_{j}, j=1, \ldots, n$. 
2. $H(x, y)$ behaves like

$$
\frac{\left(p\left(\Phi_{j}\right)\right)^{2}}{\left(x-x_{j}\right)^{2}+\left(y-y_{j}\right)^{2}}
$$

near each point $P_{j}, j=1,2, \ldots, n$, and $H(x, y)$ behaves like

$$
\frac{\left(p\left(\Phi_{1}+\Phi_{2}+\ldots+\Phi_{n}\right)\right)^{2}}{x^{2}+y^{2}}
$$

near infinity.

Under a gauge transformation $u \mapsto \phi \cdot u$ with an arbitrary smooth function $\phi: M \rightarrow U(1)$, the Dirichlet form $\mathcal{Q}_{A}[u]$ becomes $\mathcal{Q}_{A^{\prime}}[u]$ with $A^{\prime}=A+\frac{1}{i} \phi^{-1} \nabla \phi$. The right hand side of (1.7) is invariant under this gauge transform. Hence, it is sufficient to establish (1.7) for any $A$ from a given gauge equivalent class of magnetic vector potentials.

We show in this paper that any analytic function $F(z)$ on $\mathbb{C}$ with zero set $\left\{P_{1}, P_{2}, \ldots, P_{n}\right\}$ and $F(\infty)=\infty$ generates a function $H(x, y)$ with properties 1 and 2 above. It is also possible to choose an analytic function $F(z)$ which gives an $H(x, y)$ with the additional property

3. If $p\left(\Phi_{j}\right) \rightarrow 0$ for some $j$ then the "contribution" from $P_{j}$ in $H$ also goes to zero.

For the reader's convenience we finish this introduction by giving an example of $H(x, y)$ in the case of two points $P_{1}=-1$ and $P_{2}=1$ in $\mathbb{C}$ with the circulations $c_{1} \equiv \Phi_{1}$ and $c_{2} \equiv \Phi_{2}$, respectively.

Example 1.1. Let $P_{1}=(-1,0), P_{2}=(1,0)$ be two points in $\mathbb{R}^{2}, M=\mathbb{R}^{2} \backslash$ $\left\{P_{1}, P_{2}\right\}$ and $A$ is a magnetic vector potential of Aharonov-Bohm type in $M$ with the circulations $c_{j}$ round $P_{j}, j=1,2$. Then the inequality (1.7) holds with

$$
H(x, y)=C(x, y) \cdot\left|\frac{2 z}{z^{2}-1}\right|^{2}, \quad z=x+i y,
$$

where $C(x, y)$ is the piecewise constant function on $\mathbb{R}^{2}$ shown in Figure 1 . In the figure, $C$ is the curve $\left(x^{2}-y^{2}-1\right)+4 x^{2} y^{2}=1$ which divides the plane $\mathbb{R}^{2}$ into three regions $\Omega_{1}, \Omega_{2}$ and $\Omega_{\infty}$, where $P_{1} \in \Omega_{1}$ and $P_{2} \in \Omega_{2} ; C(x, y)$ equals $\left(p\left(c_{1}\right)\right)^{2}$ in $\Omega_{1},\left(p\left(c_{2}\right)\right)^{2}$ in $\Omega_{2}$ and $\left(p\left(c_{1}+c_{2}\right)\right)^{2} / 4$ in $\Omega_{\infty}$.

\section{Inequality for doubly connected domains}

Let $\Omega$ denote a bounded doubly connected domain (i.e. the boundary of $\Omega$ is a disjoint union of two closed simple curves) with a smooth boundary in the plane $\mathbb{R}^{2}=\mathbb{C}$. $\Omega$ is homeomorphic to an open annulus. Let $\Omega_{r, R}(r<R)$ be an annulus in $\mathbb{C}$ with the internal radius $r$, the external radius $R$ and with the center at the origin, i.e.

$$
\Omega_{r, R}=\{z \in \mathbb{C}|r<| z \mid<R\} .
$$

From the theory of functions of one complex variable we know (see [5, Theorem 1.2]) that any doubly connected domain can be conformally mapped onto 


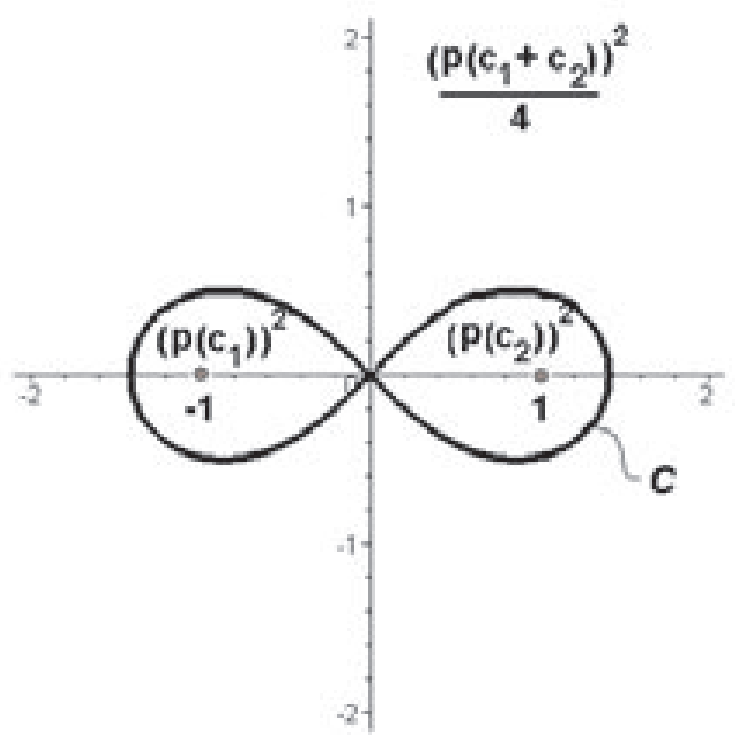

Figure 1. Function $C(x, y)$.

an annulus $\Omega_{r, R}$ for some $r$ and $R$. For a conformal mapping $F: \Omega \rightarrow \Omega_{r, R}$ let us define a function $\mathcal{B}_{\Omega, F}(x, y)$ on $\Omega$ by

$$
\mathcal{B}_{\Omega, F}(x, y)=\left|\frac{F_{z}^{\prime}(z)}{F(z)}\right|^{2}
$$

where $z=x+i y$ and $F_{z}^{\prime}$ denote the complex derivative of $F$.

Lemma 2.1. The function $\mathcal{B}_{\Omega, F}$ defined by (2.1) does not depend on the choice of the conformal mapping $F$.

Proof. Consider any other conformal mapping $\tilde{F}$ from $\Omega$ onto $\Omega_{\tilde{r}, \tilde{R}}$. From Theorem 1.3 [5] we know that

$$
\frac{R}{r}=\frac{\tilde{R}}{\tilde{r}}
$$

Hence, since the right-hand side of (2.1) is invariant under scaling $F \mapsto$ const $\cdot F$, we can assume that $r=\tilde{r}$ and $R=\tilde{R} . \tilde{F} \circ F^{-1}$ is a conformal automorphism of $\Omega_{r, R}$. Since any holomorphic automorphism of $\Omega_{r, R}$ is a composition of rotations and reflections (see p. 133 in [2]), we have to check that the right-hand side of (2.1) is invariant under $F \mapsto \mu \cdot F$ (for $\mu$ a unimodular constant) and under $F \mapsto \frac{r \cdot R}{F}$. This is clear and hence the proof is completed.

We shall use the notation $\mathcal{B}_{\Omega}$ instead of $\mathcal{B}_{\Omega, F}$.

Let $A=\left(A_{1}(x, y), A_{2}(x, y)\right)$ be a smooth magnetic vector potential in $\bar{\Omega}$ with zero magnetic field (1.2). Recall that a circulation $\Phi$ of $A$ in the doubly connected 
domain $\Omega$ is

$$
\Phi=\frac{1}{2 \pi} \oint_{\sigma} A_{1}(x, y) d x+A_{2}(x, y) d y
$$

where $\sigma$ is a closed path which parameterizes the "internal" component of the boundary of $\Omega$. The last integral is invariant under continuous deformations of $\sigma$.

Theorem 2.2. Let $\Omega$ be a bounded doubly connected domain in $\mathbb{R}^{2}$ with a smooth boundary. For any smooth function $f \in C^{\infty}(\bar{\Omega})$ we have

$$
\int_{\Omega}|(\nabla+i A) f|^{2} d x d y \geq(p(\Phi))^{2} \int_{\Omega} \mathcal{B}_{\Omega}(x, y)|f(x, y)|^{2} d x d y,
$$

where $\mathcal{B}_{\Omega}(x, y)$ is defined by $(2.1)$ and $p(\Phi)$ is defined by (1.8).

Proof. First let us prove that for $\Omega_{r, R}$ and $\tilde{A}=\frac{\Phi}{x^{2}+y^{2}}(-y, x)$ the following inequality holds

$$
\int_{\Omega_{r, R}}|(\nabla+i \tilde{A}) f|^{2} d x d y \geq(p(\Phi))^{2} \int_{\Omega_{r, R}} \frac{|f(x, y)|^{2}}{x^{2}+y^{2}} d x d y,
$$

for any $f \in C^{\infty}\left(\overline{\Omega_{r, R}}\right)$ (see [3] for more general results). The left-hand side and the right-hand side are both invariant under rotation of $\mathbb{R}^{2}$ around the origin. So, it is sufficient to establish (2.3) for spherical functions $f(r) e^{i n \theta}, n \in \mathbb{Z}$ and $r=\sqrt{x^{2}+y^{2}}$. For such functions

$$
\begin{gathered}
\int_{\Omega_{r, R}}\left|(\nabla+i \tilde{A}) f(r) e^{i n \theta}\right|^{2} d x d y=\int_{\Omega_{r, R}}\left(\left|f_{r}^{\prime}\right|^{2}+\frac{1}{r^{2}}|f(r)|^{2} \cdot(n+\Phi)^{2}\right) d x d y \\
\geq \int_{\Omega_{r, R}} \frac{1}{r^{2}}|f(r)|^{2} \cdot(n+\Phi)^{2} d x d y \geq(p(\Phi))^{2} \int_{\Omega_{r, R}} \frac{\left|f(r) e^{i n \theta}\right|^{2}}{x^{2}+y^{2}} d x d y .
\end{gathered}
$$

Now, let $F: \Omega \rightarrow \Omega_{r, R}$ be a conformal mapping, $F(x, y)=(u(x, y), v(x, y))$. Denote by $A^{F}(u, v)=\left(A_{1}^{F}(u, v), A_{2}^{F}(u, v)\right)$ a magnetic vector potential in $\Omega_{r, R}$ such that $F^{*}\left(\omega_{A^{F}}\right)=\omega_{A}$, i.e.

$$
A_{1}^{F}(u, v) d u+A_{2}^{F}(u, v) d v=A_{1}(x, y) d x+A_{2}(x, y) d y .
$$

The magnetic vector potential $A^{F}$ also has zero magnetic field and the same circulation $\Phi$ as $A$. 
Since $F$ is a conformal mapping, the reader will have no difficulty in showing that for any $f \in C^{\infty}\left(\overline{\Omega_{r, R}}\right)$ we have

$$
\begin{gathered}
\int_{\Omega_{r, R}}\left(\left|\left(\nabla_{u}+i A_{1}^{F}(u, v)\right) f(u, v)\right|^{2}+\left|\left(\nabla_{v}+i A_{2}^{F}(u, v)\right) f(u, v)\right|^{2}\right) d u d v \\
=\int_{\Omega}\left(\left|\left(\nabla_{x}+i A_{1}(x, y)\right) f(u(x, y), v(x, y))\right|^{2}\right. \\
\left.+\left|\left(\nabla_{y}+i A_{2}(x, y)\right) f(u(x, y), v(x, y))\right|^{2}\right) d x d y
\end{gathered}
$$

Since $A^{F}$ is gauge equivalent to the magnetic vector potential $\frac{\Phi}{u^{2}+v^{2}}(-v, u)$ and the inequality (2.3) is also invariant under gauge transformations, we have that

$$
\int_{\Omega}|(\nabla+i \tilde{A}) f(u(x, y), v(x, y))|^{2} d x d y \geq(p(\Phi))^{2} \int_{\Omega_{r, R}} \frac{|f(u, v)|^{2}}{u^{2}+v^{2}} d u d v .
$$

Taking into account that

$$
\int_{\Omega_{r, R}} \frac{|f(u, v)|^{2}}{u^{2}+v^{2}} d u d v=\int_{\Omega}|f(u(x, y), v(x, y))|^{2} \frac{\left|F_{z}^{\prime}\right|^{2}}{|F|^{2}} d x d y,
$$

we obtain from (2.5) the inequality (2.2). This completes the proof.

\section{Inequalities for punctured plane}

Theorem 2.2 gives us a Hardy-type inequality for a magnetic vector potential of Aharonov-Bohm type in a bounded doubly connected domain. For a more general domain $\Omega$, e.g. a Riemann surface or multiply connected domain, our strategy is:

a) find a decomposition, up to a zero measure set, of the domain $\Omega$ into doubly connected domains;

b) find conformal mappings of these doubly connected domains into annuli and apply Theorem 2.2.

The classical Morse theory (see e.g. [4]) is the most natural tool for constructing decompositions into doubly connected domains. But, in general, finding a conformal mapping from a doubly connected domain into $\Omega_{r, R}$ is a difficult problem. Our idea in this article is to use a function $|F|$ for constructing a Morse complex, where $F: \Omega \rightarrow \mathbb{C}$ is an holomorphic function. In this case, the function $|F|$ provides a decomposition and $F$ provides the conformal mappings. We are going to apply this idea in the case of the punctured plane $M=\mathbb{C} \backslash\left\{P_{1}, P_{2}, \ldots, P_{n}\right\}$ with a smooth magnetic vector potential $A$ of Aharonov-Bohm type.

Let $F: \mathbb{C} \rightarrow \mathbb{C}$ be an analytic function with zero set $\left\{P_{1}, P_{2}, \ldots, P_{n}\right\}$, i.e. $F^{-1}(0)=\left\{P_{1}, P_{2}, \ldots, P_{n}\right\}$, and $F(\infty)=\infty$. Denote by $\operatorname{ord}_{P_{j}} F$ the order of zero of $F$ at $P_{j}$. Let $\left\{Q_{1}, Q_{2}, \ldots, Q_{l}\right\}$ be a zero set of the complex derivative $F_{z}^{\prime}$ 
of the function $F$, i.e. $\left\{Q_{1}, Q_{2}, \ldots, Q_{l}\right\}=\left(F_{z}^{\prime}\right)^{-1}(0)$, and denote by Crit $_{F}$ the following subset of $\mathbb{R}_{+}=\{x \in \mathbb{R} \mid x \geq 0\}$ :

$$
\operatorname{Crit}_{F}=\left\{0,\left|F\left(Q_{1}\right)\right|, \ldots,\left|F\left(Q_{l}\right)\right|\right\} .
$$

Under the map $|F|: \mathbb{C} \rightarrow \mathbb{R}_{+}$the pre-image of $\mathrm{Crit}_{F}$ is a zero measure set $\mathcal{F}_{c}$.

Let us define a piecewise constant function $C_{F}$ on $\mathbb{R}^{2}$. For any $(x, y) \in \mathbb{R}^{2}$, $x+i y \notin \mathcal{F}_{c}$, the set $|F|^{-1}(|F|(x+i y))$ is a disjoint union of smooth simple curves in $\mathbb{C}$ and let $\gamma_{(x, y)}$ denote one of them that goes through the point $(x, y)$. This $\gamma_{(x, y)}$ divides $\mathbb{C}$ into two domains, a bounded domain $\Omega_{\text {int }}\left(\gamma_{(x, y)}\right)$ and an unbounded domain $\Omega_{\text {ext }}\left(\gamma_{(x, y)}\right)$. Then

$$
C_{F}(x, y):=\frac{\left(p\left(\sum_{P_{k} \in \Omega_{\text {int }}\left(\gamma_{(x, y)}\right)} \Phi_{k}\right)\right)^{2}}{\left(\operatorname{ord}_{\gamma_{(x, y)}} F\right)^{2}},
$$

where $\Phi_{k}$ is a circulation of $A$ round $P_{k}$ and

$$
\operatorname{ord}_{\gamma_{(x, y)}} F=\sum_{P_{k} \in \Omega_{\mathrm{int}}\left(\gamma_{(x, y)}\right)} \operatorname{ord}_{P_{k}} F
$$

We can now state our main result.

Theorem 3.1. Let $C_{F}$ be defined in (3.1) for the analytic function F. For any $u \in C_{0}^{\infty}(M)$ the following inequality holds

$$
\int_{M}|(\nabla+i A) u|^{2} d x d y \geq \int_{M} C_{F}(x, y)\left|\frac{F_{z}^{\prime}(x+i y)}{F(x+i y)}\right|^{2}|u(x, y)|^{2} d x d y
$$

Proof. Let $\mathbb{R}_{+} \backslash \operatorname{Crit}_{F}=\bigcup_{m \in \mathbb{Z}}\left[a_{m}, b_{m}\right]$ such that $\left(a_{m}, b_{m}\right) \cap\left(a_{m^{\prime}}, b_{m^{\prime}}\right)=\emptyset$ for $m \neq m^{\prime}$. From Morse theory we know that $|F|^{-1}\left(\left(a_{m}, b_{m}\right)\right)$ is a disjoint union of doubly connected domains. Let $\Omega_{0}$ be any connected component of $|F|^{-1}\left(\left(a_{m}, b_{m}\right)\right)$. Then $\left.F\right|_{\Omega_{0}}: \Omega_{0} \rightarrow \Omega_{a_{m}, b_{m}}$ is a holomorphic function from $\Omega_{0}$ onto an annulus $\Omega_{a_{m}, b_{m}}$. Since we are away from the critical set of $F$, the holomorphic map $\left.F\right|_{\Omega_{0}}$ is a covering map from $\Omega_{0}$ onto the annulus $\Omega_{a_{m}, b_{m}}$. From the Argument Principle (see, e.g. [1]) the degree of $\left.F\right|_{\Omega_{0}}$ equals to $\operatorname{ord}_{\gamma_{(x, y)}} F$ defined in (3.2), where $(x, y) \in \Omega_{0}$. Therefore the function $\left(\left.F\right|_{\Omega_{0}}\right)^{1 / \operatorname{ord}_{\gamma_{(x, y)}}{ }}$ is well defined and is a conformal mapping from $\Omega_{0}$ onto an annulus $\Omega_{r_{m}, R_{m}}$, where

$$
r_{m}=\left(a_{m}\right)^{1 / \operatorname{ord}_{\gamma_{(x, y)}} F} \quad \text { and } \quad R_{m}=\left(b_{m}\right)^{1 / \operatorname{ord}_{\gamma_{(x, y)}} F} .
$$


From Theorem 2.2

$$
\begin{aligned}
& \int_{\Omega_{0}}|(\nabla+i A) u|^{2} d x d y \\
& \quad \geq \int_{\Omega_{0}}\left(p\left(\sum_{P_{k} \in \Omega_{\text {int }}\left(\gamma_{(x, y)}\right)} \Phi_{k}\right)\right)^{2} \mid \frac{\left(\left(\left.F\right|_{\Omega_{0}}\right)^{\left.1 / \operatorname{ord}_{\gamma_{(x, y)} F}\right)_{z}^{\prime}}\right.}{\left.\left(\left.F\right|_{\Omega_{0}}\right)^{1 / \operatorname{ord}_{(x, y)} F}\right|^{2}|u|^{2} d x d y} \\
& \quad=\int_{\Omega_{0}} C_{F}(x, y)\left|\frac{F_{z}^{\prime}(x+i y)}{F(x+i y)}\right|^{2}|u(x, y)|^{2} d x d y .
\end{aligned}
$$

Summing (3.4) over all connected component of $|F|^{-1}\left(\left(a_{m}, b_{m}\right)\right)$ and over all $m \in \mathbb{Z}$, we obtain Theorem 3.1. This conclude the proof.

Remark 3.2. Choosing $F(z)=\prod_{j=1}^{n}\left(z-z_{j}\right)$ we obtain a function

$$
H_{F}(x, y)=C_{F}(x, y)\left|\frac{F_{z}^{\prime}(x+i y)}{F(x+i y)}\right|^{2}
$$

which satisfies the conditions 1 and 2 from the Introduction. Choosing

$$
F(z)=\frac{1}{\sum_{j=1}^{n} \frac{p\left(\Phi_{j}\right)}{\left(z-z_{j}\right)}}
$$

gives $H_{F}(x, y)$ with the additional property 3 from the Introduction.

\section{Acknowledgement}

The author is grateful to W. D. Evans for useful discussions.

\section{References}

[1] L. V. Ahlfors, Complex analysis. An introduction to the theory of analytic functions of one complex variable, Third edition. International Series in Pure and Applied Mathematics. McGraw-Hill Book Co., New York, 1978.

[2] S. Krantz, Complex analysis: the geometric viewpoint, Carus Mathematical Monographs, 23, Mathematical Association of America, Washington, DC, 1990.

[3] A. Laptev, T. Weidl, Hardy inequalities for magnetic Dirichlet forms, Mathematical results in quantum mechanics (Prague, 1998), 299-305, Oper. Theory Adv. Appl., 108, Birkhäuser, Basel, 1999.

[4] J. Milnor, Morse theory, Annals of Mathematics Studies, No. 51. Princeton University Press, Princeton, NJ, 1963

[5] G.-C. Wen, Conformal mappings and boundary value problems, Translations of Mathematical Monographs, 106. American Mathematical Society, Providence, RI, 1992.

School of Mathematics, Cardiff University, 23 Senghennydd Road, P.O. Box 926, Cardiff CF24 4YH, Wales, Great Britain.

E-mail address: BalinskyA@cardiff.ac.uk 